Redistribution of pump power and impairments in gain-equalized distributed fiber Raman amplifiers due to four-wave mixing and parametric amplification

This article has been downloaded from IOPscience. Please scroll down to see the full text article.

2008 J. Opt. A: Pure Appl. Opt. 10104004

(http://iopscience.iop.org/1464-4258/10/10/104004)

View the table of contents for this issue, or go to the journal homepage for more

Download details:

IP Address: 128.178.23.47

The article was downloaded on 01/11/2012 at 14:23

Please note that terms and conditions apply. 


\title{
Redistribution of pump power and impairments in gain-equalized distributed fiber Raman amplifiers due to four-wave mixing and parametric amplification
}

\author{
Marcelo A Soto and Ricardo Olivares \\ Department of Electronics, Universidad Técnica Federico Santa María, Casilla 110-V, \\ Valparaíso, Chile \\ E-mail: ricardo.olivares@usm.cl
}

Received 7 March 2008, accepted for publication 28 May 2008

Published 27 August 2008

Online at stacks.iop.org/JOptA/10/104004

\begin{abstract}
In this work, by using a comprehensive numerical model which rigorously describes the interaction between stimulated Raman scattering (SRS) and four-wave mixing (FWM), we verify that FWM processes, including depletion and parametric gain, generate a redistribution of pump power in distributed fiber Raman amplifiers (DFRAs). As a consequence of pump-pump FWM, several FWM components can be generated, which act as new sources of SRS for Raman pumping. Due to new SRS-FWM interactions, a redistribution and exchange of pump power along the fiber also occurs, producing degradation in the performance of the amplifier. Numerical results show impairments in distributed amplified systems due to these interactions, such as loss of flatness on the spectral gain, reduction on the net Raman gain, and the presence of strong FWM products within the transmission band. We note that the localization of the zero dispersion wavelength $\left(\lambda_{\mathrm{ZD}}\right)$ of the fiber is a critical factor in the occurrence of these impairments. A reduction of net Raman gain up to $3 \mathrm{~dB}$ and tilt up to $7 \mathrm{~dB}$ in the spectral gain profile have been found in different amplified systems as consequence of pump-pump FWM and parametric gain of Raman pumps.
\end{abstract}

Keywords: distributed fiber Raman amplifiers, four-wave mixing, parametric amplification

\section{Introduction}

Recent investigations have demonstrated the efficient amplification provided by distributed fiber Raman amplifiers (DFRAs) in ultra-broadband long-haul transmission systems [1]. DFRAs are characterized by having ultrawide bandwidth, flexible wavelength operation, low noise, and capacity to mitigate fiber nonlinearities. Nevertheless, the expected performance of a multi-pumped DFRA for broadband systems is mainly determined by the efficiency and rigorousness of the method used to design it $[1,2]$. Several techniques have been proposed to optimize the spectral gain profile of a DFRA in order to provide a flat gain for all the wavelength division multiplexed (WDM) channels in the system [2-6]. The objective of all these methods is to find both the optimum spectral position and the input power of every pump of the amplifier. However, the main drawback is that the mathematical models used in these approaches do not consider the four-wave mixing (FWM) [7, 8] interaction between pumps which can generate an important degradation in the system performance. For instance, if the designed amplifier is implemented in a fiber with low chromatic dispersion in the region where the pump are located, pump-pump FWM and parametric amplification of the pumps could be produced [9]. As a consequence, redistribution and exchange of pump power along the fiber could occur, and FWM components acting as new sources for Raman pumping might take place. All this could result in a distorted signal gain spectrum and degraded optical signal-to-noise ratio (OSNR) [10].

FWM interactions in DFRAs have been studied by other authors [10-12], but the models that they have used ignore 
pump depletion and parametric gain or consider stimulated Raman scattering (SRS) and FWM processes separately, using analytical approximations [10] or iterative numerical methods [11]. In this paper we use a novel and comprehensive numerical model to analyze how the propagation of Raman pumps is affected along the amplifier through FWM and parametric amplification processes [13], which solves the interaction of SRS and FWM in a straightforward way. By simulating cases where co-propagating Raman pumps are located near the zero dispersion wavelength $\left(\lambda_{\mathrm{ZD}}\right)$ we show a redistribution and exchange of pump power produced by the parametric processes involved, and we demonstrate how they affect the net Raman gain for different configurations of flatgain multi-pumped DFRAs.

\section{Theoretical model}

In the model, the complex envelope of all electric fields is normalized and expressed as $A_{F}(z)$ (where $F$ is related to the propagation frequency $f_{F}$ ) such that the optical power is conveniently described as $P_{F}(z)=\left|A_{F}(z)\right|^{2}[10]$. The mathematical model used here to describe the interaction of SRS and FWM processes in a co-propagating Raman pump configuration can be derived from the expressions given in [3-8]. It describes the propagation of a lightwave at frequency $f_{F}$ in direction $+z$, which interacts by SRS with other waves at different frequencies $f_{j}$. When $f_{j}>f_{F}$ the model presents the Raman amplification of the wave at $f_{F}$, while $f_{j}<f_{F}$ implies that the wave at $f_{F}$ is depleted due to SRS. The model also considers waves at frequencies $f_{l}, f_{k}$, and $f_{m}$ which produce FWM products or parametric gain at frequency $f_{F}$. Finally, the depletion of the wave at $f_{F}$ due to FWM when interacting with lightwaves at $f_{p}, f_{q}$, and $f_{r}$ is also described. Thus, the model becomes

$$
\begin{aligned}
& \frac{\mathrm{d} A_{F}(z)}{\mathrm{d} z}=-\frac{\alpha_{F}}{2} A_{F}(z) \\
& \quad+\sum_{f_{j}>f_{F}} \frac{g_{\mathrm{R}}\left(f_{F}, f_{j}\right)}{2 A_{\mathrm{eff}} K_{\mathrm{eff}}\left(f_{F}, f_{j}\right)}\left|A_{j}(z)\right|^{2} A_{F}(z) \\
& \quad-\sum_{f_{j}<f_{F}} \frac{f_{F}}{f_{j}} \frac{g_{\mathrm{R}}\left(f_{F}, f_{j}\right)}{2 A_{\mathrm{eff}} K_{\mathrm{eff}}\left(f_{F}, f_{j}\right)}\left|A_{j}(z)\right|^{2} A_{F}(z) \\
& +\mathrm{i} \frac{1}{3} \gamma \sum_{k, l \neq m}\left\{K_{f w m}\left(f_{k}, f_{l}, f_{m}\right) D_{k l m}\right. \\
& \left.\quad \times A_{k}(z) A_{l}(z) A_{m}^{*}(z) \exp \left(\mathrm{i} \Delta \beta_{k l m} z\right)\right\} \\
& +\mathrm{i} \frac{1}{3} \gamma \sum_{p, r \neq q}\left\{K_{f w m}\left(f_{F}, f_{q}, f_{r}\right) D_{p r q}\right. \\
& \left.\quad \times A_{p}(z) A_{q}^{*}(z) A_{r}(z) \exp \left(-\mathrm{i} \Delta \beta_{p r q} z\right)\right\}
\end{aligned}
$$

where $\alpha_{F}$ is the attenuation coefficient of the fiber at frequency $f_{F}, A_{\text {eff }}$ is the effective area, $g_{\mathrm{R}}$ is the Raman gain coefficient, $\gamma$ is the nonlinear coefficient, $D_{k l m(p r q)}$ is the degeneracy factor, and $\Delta \beta_{k l m(p r q)}$ is the linear phasemismatch, so $\Delta \beta_{k l m(p r q)}=\beta_{k(p)}+\beta_{l(r)}-\beta_{m(q)}-\beta_{F}$ [9]. $K_{\text {eff }}$ and $K_{f w m}$ are the polarization factors for SRS and FWM, respectively. $K_{\text {eff }}$ is 0.5 or 1 , depending on whether the waves at frequency $f_{F}$ and $f_{j}$ have identical or random polarization states, respectively [3]. $K_{f w m}$ is 1 when the waves involved in the FWM process have parallel linear polarizations, and $(1 / 2)^{0.5}$ or $(3 / 8)^{0.5}$ for partially degenerate and non-degenerate FWM with random polarization states, respectively [14].

The first term on the right-hand side of (1) represents the fiber attenuation. The second and third terms describe the gain and depletion due to stimulated Raman scattering, respectively. The fourth term describes the contribution of FWM products and the parametric gain to $A_{F}(z)$ obtained from all the combinations of electric fields at frequencies $f_{l}, f_{k}$, and $f_{m}$ (with $l, k \neq m$ ) that satisfy the relation $f_{F}=f_{k}+f_{l}-f_{m}$. The fifth term describes the depletion of $A_{F}(z)$ due to FWM processes for all the frequency combinations $f_{p}, f_{q}$, and $f_{r}$ (with $F, q \neq r$ ) where the condition $f_{p}=f_{F}+f_{q}-f_{r}$ is satisfied (here $A_{F}(z)$ acts as a pump of the FWM process).

Equation (1) provides a simultaneous description of Raman gain/depletion, parametric gain, depletion due to FWM, and generation of FWM waves. This complete and simultaneous description of both nonlinearities allows for solving the model by a simple numerical method. Therefore, it is not required to use any iterative method to get convergence in the solution (iterative methods are required when SRS and FWM models are solved separately) [11, 12], while ultimately reducing the computing time. It is also a good alternative instead of using closed mathematical expressions based on undepleted conditions for the waves [10], which can be inappropriate approximations when a strong interaction between SRS and FWM exists.

\section{Numerical solution of the model}

The proposed model describes the propagation of electric field waves in terms of a system of complex, nonlinear and coupled differential equations. If $N$ signals with different wavelengths, including pumps and WDM channels, are introduced into the fiber, $\left(N^{3}-N^{2}\right) / 2$ FWM products will be generated. Therefore, in the worst case, a system of $\left(N^{3}-N^{2}\right) / 2+N$ differential equations, each one similar to (1), will be obtained (one for each lightwave frequency). This system of equations can be solved by a simple numerical technique. In this work, we have used the fourth-order Runge-Kutta method to solve it, separating (1) into two parts: one related to the amplitude of the electric field $\left(\left|A_{F}(z)\right|\right)$, and the other associated with its phase $\left(\phi_{F}(z)\right)$. Thus, from (1) the following expressions are obtained:

$$
\begin{aligned}
& \frac{\mathrm{d}\left|A_{F}(z)\right|}{\mathrm{d} z}=-\frac{\alpha_{F}}{2}\left|A_{F}(z)\right| \\
& \quad+\sum_{f_{j}>f_{F}} \frac{g_{\mathrm{R}}\left(f_{F}, f_{j}\right)}{2 A_{\mathrm{eff}} K_{\mathrm{eff}}\left(f_{F}, f_{j}\right)}\left|A_{j}(z)\right|^{2}\left|A_{F}(z)\right| \\
& \quad-\sum_{f_{j}<f_{F}} \frac{f_{F}}{f_{j}} \frac{g_{\mathrm{R}}\left(f_{F}, f_{j}\right)}{2 A_{\mathrm{eff}} K_{\mathrm{eff}}\left(f_{F}, f_{j}\right)}\left|A_{j}(z)\right|^{2}\left|A_{F}(z)\right| \\
& \quad-\frac{1}{3} \gamma \sum_{k, l \neq m}\left\{K_{f w m}\left(f_{k}, f_{l}, f_{m}\right) D_{k l m}\right. \\
& \left.\quad \times\left|A_{k}(z) \| A_{l}(z)\right|\left|A_{m}(z)\right| \sin \left[\Delta \beta_{k l m} z+\phi_{k l m}(z)\right]\right\} \\
& \quad-\frac{1}{3} \gamma \sum_{p, r \neq q}\left\{K_{f w m}\left(f_{F}, f_{q}, f_{r}\right) D_{p r q}\right. \\
& \left.\quad \times\left|A_{p}(z)\right|\left|A_{q}(z) \| A_{r}(z)\right| \sin \left[-\Delta \beta_{p r q} z+\phi_{p r q}(z)\right]\right\}
\end{aligned}
$$




$$
\begin{aligned}
& \frac{\mathrm{d} \phi_{F}(z)}{\mathrm{d} z}=\frac{1}{3} \gamma \sum_{k, l \neq m}\left\{K_{f w m}\left(f_{k}, f_{l}, f_{m}\right) D_{k l m}\right. \\
& \left.\quad \times \frac{\left|A_{k}(z)\right|\left|A_{l}(z)\right|\left|A_{m}(z)\right|}{\left|A_{F}(z)\right|} \cos \left[\Delta \beta_{k l m} z+\phi_{k l m}(z)\right]\right\} \\
& \quad+\frac{1}{3} \gamma \sum_{p, r \neq q}\left\{K_{f w m}\left(f_{F}, f_{q}, f_{r}\right) D_{p r q}\right. \\
& \left.\quad \times \frac{\left|A_{p}(z)\right|\left|A_{q}(z)\right|\left|A_{r}(z)\right|}{\left|A_{F}(z)\right|} \cos \left[-\Delta \beta_{p r q} z+\phi_{p r q}(z)\right]\right\}
\end{aligned}
$$

where

$$
\begin{aligned}
& \phi_{k l m}(z)=\phi_{k}(z)+\phi_{l}(z)-\phi_{m}(z)-\phi_{F}(z) \\
& \phi_{p r q}(z)=\phi_{p}(z)+\phi_{r}(z)-\phi_{q}(z)-\phi_{F}(z) .
\end{aligned}
$$

The effect of FWM on the amplitude and phase of the propagated lightwaves can be observed in (2) and (3), respectively. Meanwhile, absorption losses and SRS only affect the amplitude of the electric field.

\section{Efficiency of FWM}

To understand the exchange of power process among Raman pumps produced in a DFRA, due to pump-pump FWM processes and parametric amplification, we need to analyze the efficiency of the product terms generated through FWM in an amplifier. The well-known FWM efficiency is given by [8]

$$
\eta=\frac{\alpha^{2}}{\alpha^{2}+\Delta \beta^{2}}\left\{1+\frac{4 \mathrm{e}^{-\alpha L} \sin ^{2}(\Delta \beta L / 2)}{[1-\exp (-\alpha L)]^{2}}\right\}
$$

where $\alpha$ is the attenuation coefficient, $L$ is the fiber length, and $\Delta \beta$ is the linear phase-mismatch factor. Taking into account the frequencies involved in the FWM process $\left(f_{k}, f_{l}\right.$, and $\left.f_{m}\right)$, the chromatic dispersion $\left(D_{\mathrm{c}}\right)$ and dispersion slope $\left(S_{\mathrm{c}}\right)$ of the fiber at a given reference frequency $\left(f_{0}=c / \lambda_{0}\right), \Delta \beta$ can be obtained as follows:

$$
\begin{aligned}
\Delta \beta & =\frac{2 \pi \lambda_{0}^{2}}{c}\left[\left(f_{k}-f_{m}\right)\left(f_{l}-f_{m}\right)\right] D_{\mathrm{c}}\left(f_{0}\right) \\
& -\frac{\pi \lambda_{0}^{4}}{c^{2}}\left[\left(f_{k}-f_{m}\right)\left(f_{l}-f_{m}\right)\right]\left[f_{k}+f_{l}-2 f_{0}\right] S_{\mathrm{c}}\left(f_{0}\right)
\end{aligned}
$$

where $c$ is the speed of light. In particular, in low chromatic dispersion regions, equation (7) can be reduced as

$$
\Delta \beta=-\frac{\pi \lambda_{0}^{4}}{c^{2}}\left[\left(f_{k}-f_{m}\right)\left(f_{l}-f_{m}\right)\right]\left[f_{k}+f_{l}-2 f_{0}\right] S_{\mathrm{c}}\left(f_{0}\right)
$$

where the reference frequency corresponds to the frequency with zero chromatic dispersion $\left(f_{0}=c / \lambda_{\mathrm{ZD}}\right)$. By using the definition of equivalent frequency separation $\left(\Delta f_{k l m}\right)$ presented in [8], and defining the spectral separation of $f_{0}$ with respect to the middle frequency between $f_{k}$ and $f_{l}$ as $\Delta f_{M 0}$, we have

$$
\Delta \beta=-\frac{2 \pi \lambda_{0}^{4}}{c^{2}} \Delta f_{k l m}^{2} \cdot \Delta f_{M 0} \cdot S_{\mathrm{c}}\left(f_{0}\right)
$$

where

$$
\Delta f_{k l m}=\sqrt{\left|f_{k}-f_{m}\right|\left|f_{l}-f_{m}\right|}
$$

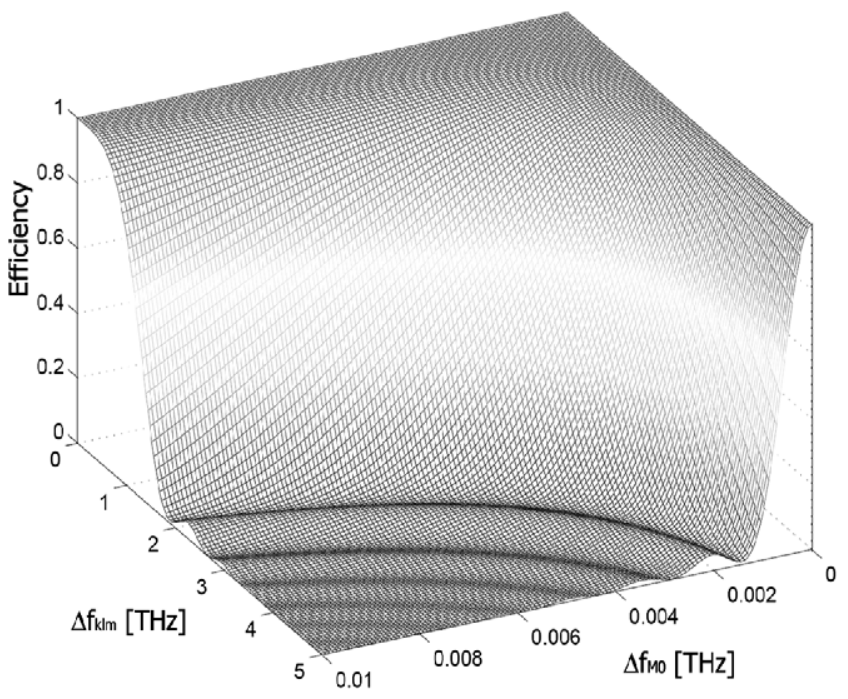

Figure 1. FWM efficiency as a function of $\Delta f_{k l m}$ and $\Delta f_{M 0}$.

and

$$
\Delta f_{M 0}=\frac{f_{k}+f_{l}}{2}-f_{0} .
$$

Figure 1 depicts the FWM efficiency as a function of $\Delta f_{k l m}$ and $\Delta f_{M 0}$, as is described by equation (6). Considering that $f_{k}, f_{l}$, and $f_{m}$ are three Raman pumps of a DFRA, there are two ways to get a strong pump-pump FWM interaction $(\eta=1)$. The first one is when $\Delta f_{k l m}=0$; however, this case needs to satisfy the condition $f_{k}=f_{m}$ or $f_{l}=f_{m}$, which correspond to self-phase modulation (SPM) and crossphase modulation (XPM) cases. The second case occurs when the condition $\Delta f_{M 0}=0$ is satisfied. For degenerated FWM cases, it occurs when the degenerated pump coincides with $\lambda_{\text {ZD }}$ $\left(f_{k}=f_{l}=c / \lambda_{\mathrm{ZD}}\right)$. However, for non-degenerate FWM cases, that condition occurs when $c / \lambda_{\text {ZD }}$ coincides with the frequency placed in the middle of the pumps, $f_{k}$ and $f_{l}$, of the process $\left(c / \lambda_{\mathrm{ZD}}=\left(f_{k}+f_{l}\right) / 2\right)$.

The generated waves resulting from FWM processes depend on the pump power levels and the efficiency of every process. Due to the high level of pump power propagating into the fiber, strong pump-pump FWM components can be obtained even if the efficiency is not exactly equal to 1 .

From both conditions presented above, note that, for broadband DFRAs, it is difficult to satisfy $\Delta f_{k l m} \approx 0$, because Raman pumps are usually distributed in a broad spectral band. However, $\Delta f_{M 0} \approx 0$ could easily occur depending on $\lambda_{\mathrm{ZD}}$ and the bandwidth of the system, which determines the number of Raman pumps. Note that this condition is independent of the spectral spacing of the pumps. Analyzing these two conditions, we can deduce that depending on the position of $\lambda_{\text {ZD }}$ within the pump band of a DFRA, it is possible that at least one high efficient pump-pump FWM product can be generated. For instance, in a DFRA with $N$ pumps, there are $N(N-1) / 2$ possible $\lambda_{\text {ZD }}$ which will cause efficient non-degenerate FWM processes $(\eta=1)$, and $N$ possibilities to produce degenerated FWM. From all these possibilities, when $\lambda_{\text {ZD }}$ coincides with a Raman pump, $N-1$ new degenerated FWM waves will be produced, while $N-2$ new FWM components will be produced 


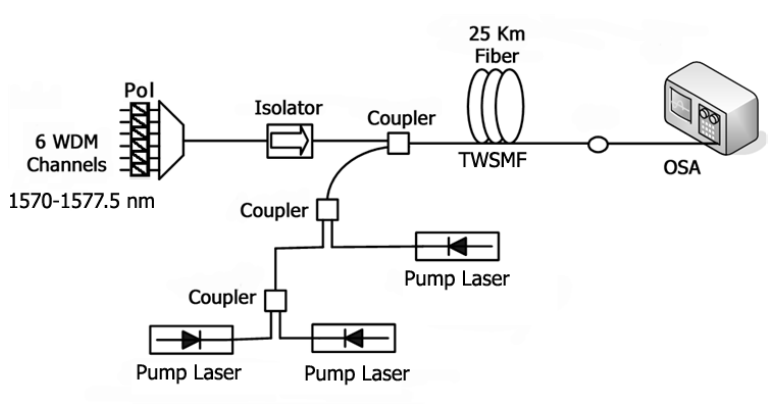

Figure 2. Experimental setup.

from non-degenerate FWM. However, different effects will be caused in a given amplified system depending on the spectral position and power of the pumps involved in an efficient FWM process.

Additionally, from equation (6) it is possible to realize that the efficiency has an oscillatory behavior along the fiber because of the factor $\sin ^{2}(\Delta \beta L / 2)$ [15]. So, if there is not a complete phase matching $(\Delta \beta \neq 0)$ the new lightwave produced by FWM will arise from noise at the fiber input $(z=0)$ and increase until it reaches its maximum at a given distance $z=L_{\mathrm{coh}}$, defined as the coherence length. Then, between $z=L_{\text {coh }}$ and $2 L_{\text {coh }}$, the FWM product will give back its energy to the pumps of the respective FWM process. The coherence length is defined as

$$
L_{\mathrm{coh}}=\frac{\pi}{\Delta \beta} .
$$

This parameter is very important for understanding how pump-pump FWM in a DFRA produces an oscillatory redistribution and exchange of power between the Raman pump and FWM components [15]. It also allows for explaining the longitudinal oscillation produced on the intensity of Raman pumps due to parametric amplification and the FWM (generation/depletion) process.

\section{Experimental results}

In order to show the non-negligible presence of pump-pump FWM in a co-pumped distributed Raman amplifier, we set up the experiment depicted in figure 2. It is composed of three depolarized pump lasers at 1450, 1480, and $1488 \mathrm{~nm}$, with 100,180 , and $150 \mathrm{~mW}$ input power, respectively (these power values consider coupler and connector losses, i.e. they are the pump powers really launched at the fiber input). The DFRA amplifies six lineally polarized WDM channels, in the range $1570-1577.5 \mathrm{~nm}(1 \mu \mathrm{W} /$ channel input power), along $25 \mathrm{~km}$ of TrueWave fiber (TWSMF) with $\lambda_{\mathrm{ZD}}=1498 \mathrm{~nm}, A_{\mathrm{eff}}=$ $55 \mu \mathrm{m}^{2}, S_{\mathrm{c}}=0.05 \mathrm{ps} \mathrm{km}^{-1} \mathrm{~nm}^{-2}$ and $\gamma=0.003 \mathrm{~W}^{-1} \mathrm{~m}^{-1}$. The fiber has been characterized in terms of Raman gain and attenuation coefficient, which have been measured as a function of the wavelength, resulting in being similar to those presented in [16]. All these parameters are used to solve a system of equations (similar to (1)) which describes the experiment.

Figure 3 shows the power spectrum at the fiber output (simulated results and measurements obtained from the optical

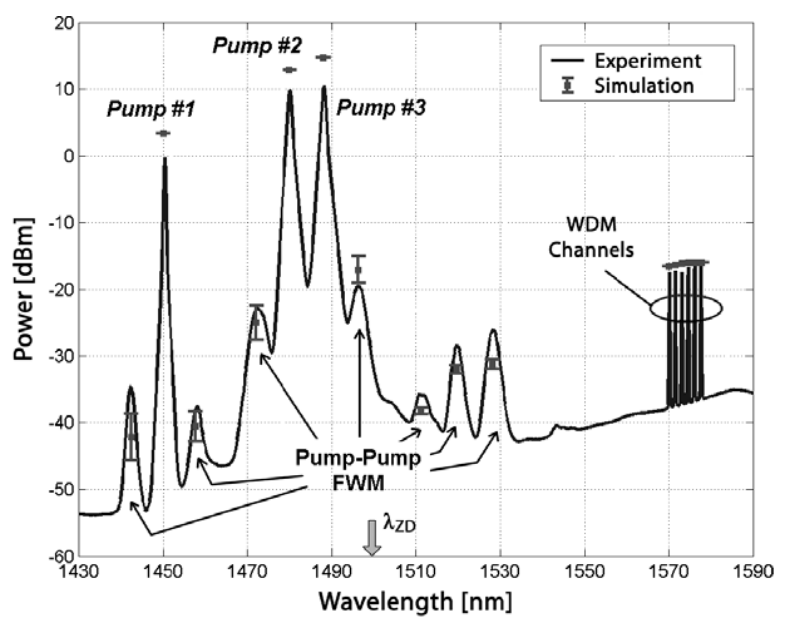

Figure 3. Experimental results.

Table 1. Designed WDM systems.

\begin{tabular}{llll}
\hline & DFRA\#1 & DFRA\#2 & DFRA\#3 \\
\hline $\begin{array}{l}\text { No. of channels } \\
\text { Band (nm) }\end{array}$ & 20 & 40 & 40 \\
\hline
\end{tabular}

spectrum analyzer (OSA)). We can observe the strong pumppump FWM processes and FWM-SRS interactions that occurred due to the low chromatic dispersion of the fiber. The new lightwaves produced by FWM cause a redistribution of the pump power, and could act as pumps for the DFRA. The simulated results are similar to the experimental measurements. The differences $(<2 \mathrm{~dB}$ on average) can be attributed mainly to possible longitudinal random fluctuations of some parameters along the fiber, such as effective area and chromatic dispersion (note the high sensitivity of the FWM process with respect to $A_{\text {eff }}$ and $\lambda_{\text {ZD }}$ for low dispersion regions).

\section{Pump-pump four-wave mixing in gain-equalized distributed Raman amplifiers}

In order to analyze the effects produced by parametric gain and FWM on the propagation of co-propagating Raman pumps along a DFRA, three co-pumped WDM systems were designed with flat gain by using the optimization method described in [5]. The objective of this method is to find the wavelength and power of each Raman pump in a DFRA, aiming at compensating the fiber attenuation, and providing a flat output power spectrum for all WDM channels involved. The optimization problem is divided into two parts, which are solved sequentially [5]. First, using a genetic algorithm it is possible to find the wavelength of each Raman pump, and then, the optimum input pump powers are found by means of an iterative method [5].

The three designed transmission systems are composed of $50 \mathrm{~km}$ of TWSMF and depolarized WDM channels with $0.5 \mathrm{~mW} /$ channel input power. The characteristics of the different amplified systems are shown in table 1 . 

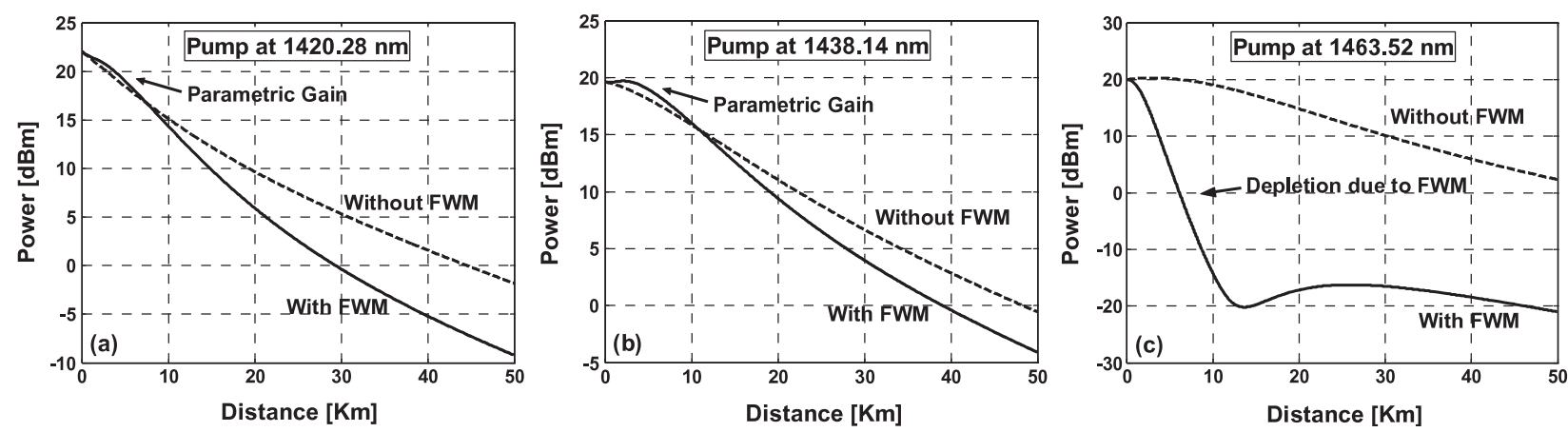

Figure 4. Longitudinal propagation of pump power on DFRA\#1, when $\lambda_{\mathrm{ZD}}=1463.52 \mathrm{~nm}$. (a) Pump at $1420.28 \mathrm{~nm}$. (b) Pump at $1438.14 \mathrm{~nm}$. (c) Pump at $1463.52 \mathrm{~nm}$.

Table 2. Optimized pumps for different DFRA configurations.

\begin{tabular}{llllllll}
\hline DFRA\#1 & $\lambda(\mathrm{nm})$ & 1420.28 & 1438.14 & 1463.52 & - & - & - \\
& Power (mW) & 160.93 & 92.24 & 99.61 & - & - & - \\
DFRA\#2 & $\lambda(\mathrm{nm})$ & 1420.12 & 1437.87 & 1453.41 & 1474.30 & - & - \\
& Power (mW) & 333.86 & 120.01 & 79.04 & 30.00 & - & - \\
DFRA\#3 & $\lambda(\mathrm{nm})$ & 1404.80 & 1420.10 & 1431.72 & 1444.41 & 1466.72 & 1490.05 \\
& Power $(\mathrm{mW})$ & 53.43 & 144.58 & 113.36 & 60.93 & 34.38 & 16.77 \\
\hline
\end{tabular}

For each amplifier, a ripple of $0.21,0.36$ and $0.84 \mathrm{~dB}$ was achieved by the optimization method making use of 3,4 , and 6 Raman pumps, respectively. Optimum pump powers and wavelengths for all three amplifiers are shown in table 2 .

Due to the low chromatic dispersion of the TWSMF around pump wavelengths and high pump powers, we expect that strong pump-pump FWM interactions will be produced, generating new lightwaves and causing a redistribution of the pump power.

From equations (6), (9), and (11) we can note that several positions of the $\lambda_{\mathrm{ZD}}$ will produce at least one FWM process with unitary efficiency. These positions correspond to those where $\Delta f_{M 0}=0$, which occurs when $f_{k}=f_{l}=c / \lambda_{\mathrm{ZD}}$ for degenerated FWM, and $c / \lambda_{\mathrm{ZD}}=\left(f_{k}+f_{l}\right) / 2$ for nondegenerate FWM processes. Thus, according to the previously presented theory of FWM efficiency (section 4), 6, 10, and 21 different positions of the $\lambda_{\text {ZD }}$ will produce at least one FWM process with $\eta=1$, in DFRA\#1, DFRA\#2, and DFRA\#3, respectively. However, different effects will be produced in a given amplifier depending on the dispersion properties (position of $\lambda_{\mathrm{ZD}}$ ) and characteristics of the transmission system (e.g. Raman pump levels, bandwidth, etc).

\subsection{Analysis of DFRA\#1}

In a DFRA pumped by three Raman sources, two highly efficient FWM waves are produced when $\lambda_{\text {ZD }}$ coincides with one of the pump wavelengths (degenerated FWM process). For example, in this amplifier, if $\lambda_{\mathrm{ZD}}=1463.52 \mathrm{~nm}$ the two lightwaves generated through FWM with the longest wavelength will have an efficiency $\eta=1$. In figure 4 , the propagation along the fiber of the three Raman pumps of the amplifier, when FWM and parametric amplification are undertaken, is compared to the case when these nonlinearities are not considered. In figures $4(\mathrm{a})$ and (b) it is possible to see the parametric amplification of the pumps at 1420.28 and $1438.14 \mathrm{~nm}$ along the first kilometers of fiber, as well as the strong depletion of the pump at $1463.52 \mathrm{~nm}$, shown in figure 4(c). However, at distances longer than the coherent length, Raman scattering becomes more relevant than FWM. Thus, the generated waves at 1489.81 and $1509.48 \mathrm{~nm}$, resulting from FWM, are strongly amplified by SRS due to their spectral position and high efficiency, as shown figure 5(a). Hence, an additional depletion of the pumps is produced as a consequence of the Raman amplification of these two FWM components. Figure 5(b) depicts the output power spectrum of the amplifier; note that the three Raman pumps have lower output power than when FWM and parametric amplification are not considered. As a consequence, this pump depletion affects the total Raman gain of the amplifier, as shown figure $5(\mathrm{c})$. On average, the redistribution of pump power produces a penalty of $\sim 3 \mathrm{~dB}$ in the net Raman gain.

On the other hand, when $\lambda_{\mathrm{ZD}}$ is located at $1441.58 \mathrm{~nm}$, which corresponds to the frequency in the middle of the pump band, one non-degenerate FWM component at $1445.03 \mathrm{~nm}$ is produced, as shown in figure 6(a). Due to its high power and spectral position, it pumps the WDM channels, affecting the designed power equalization. Figure 6(b) shows how the equalized condition for the output power spectrum is completely altered due to pump-pump FWM and FWM-SRS interactions. It can be noted that more than $7.0 \mathrm{~dB}$ of ripple is obtained when FWM is undertaken.

\subsection{Analysis of DFRA\#2}

Note that an interesting situation occurs when $\lambda_{\mathrm{ZD}}=$ $1428.94 \mathrm{~nm}$, where two FWM waves are produced with unitary efficiency as result of two non-degenerate FWM processes. In this case, the pumps at 1420.12 and $1437.87 \mathrm{~nm}$ are depleted because they transfer power mainly to the two 

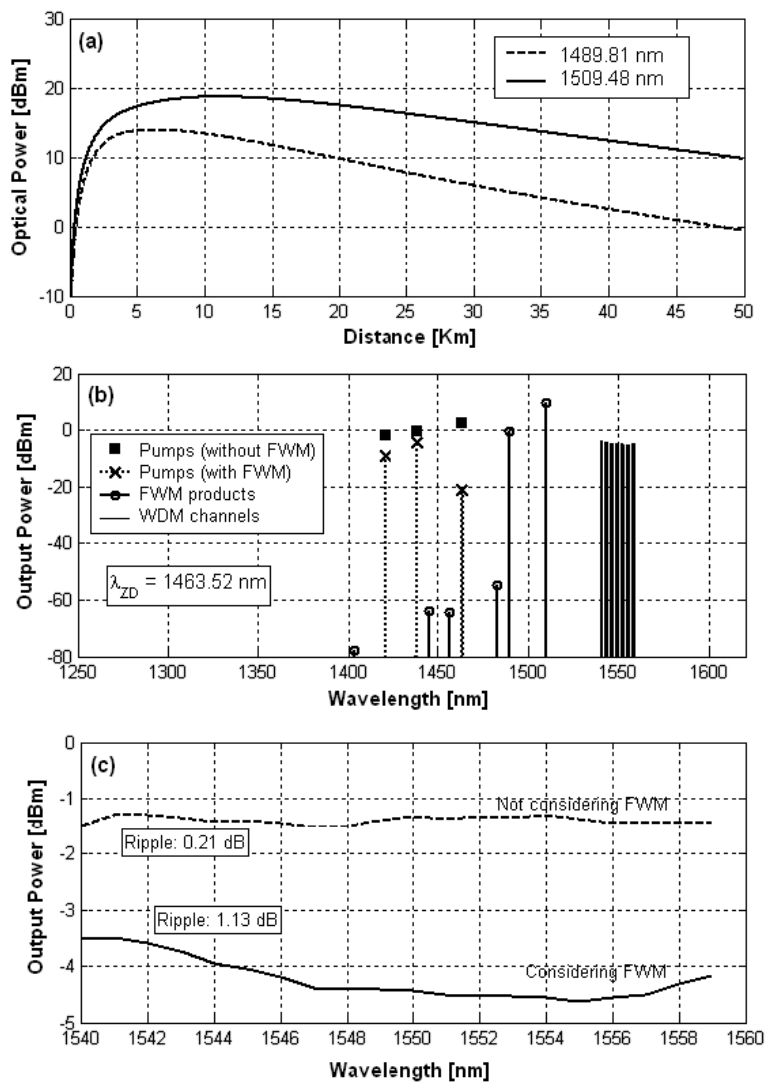

Figure 5. Output power of DFRA\#1, when $\lambda_{\mathrm{ZD}}=1463.52 \mathrm{~nm}$. (a) Most efficient FWM signals. (b) Output spectrum, including pumps, FWM products, and channels. (c) Output power for WDM channels (with and without including FWM and parametric amplification processes).

FWM products with unitary efficiency and to the pumps at 1453.41 and $1474.30 \mathrm{~nm}$. As consequence, these last two pumps are amplified through parametric gain along the first kilometers of fiber. Thus, the power of pumps and efficient FWM waves show an oscillatory propagation along the first kilometers, as shown in figures 7 and 8(a). As we can note, all this exchange of power occurs spectrally and spatially along the fiber, affecting the transfer of energy from Raman pumps to WDM channels. Hence, the Raman gain initially designed is completely modified. Thus, WDM channels with shortest wavelengths are affected by lower Raman gain, while channels with the longest wavelengths are strongly amplified by SRS. Figure 8(a) depicts the FWM signals with highest efficiency, while figure $8(\mathrm{~b})$ shows the output power spectrum of the amplified system. Note that after $50 \mathrm{~km}$ of FWM-SRS interactions, the power levels of all four pumps are lower than cases when FWM is not taken into account. This is due to two reasons: first, the two pumps with the lowest wavelengths are depleted as a consequence of FWM and parametric amplification, and second, the other two pumps are additionally depleted due to SRS. Note that as the pump power increases (in this case as a consequence of parametric amplification), the pump depletion also increases as a consequence of higher transfer of energy. Figure 8(c) depicts a comparison between the equalized output power spectrum
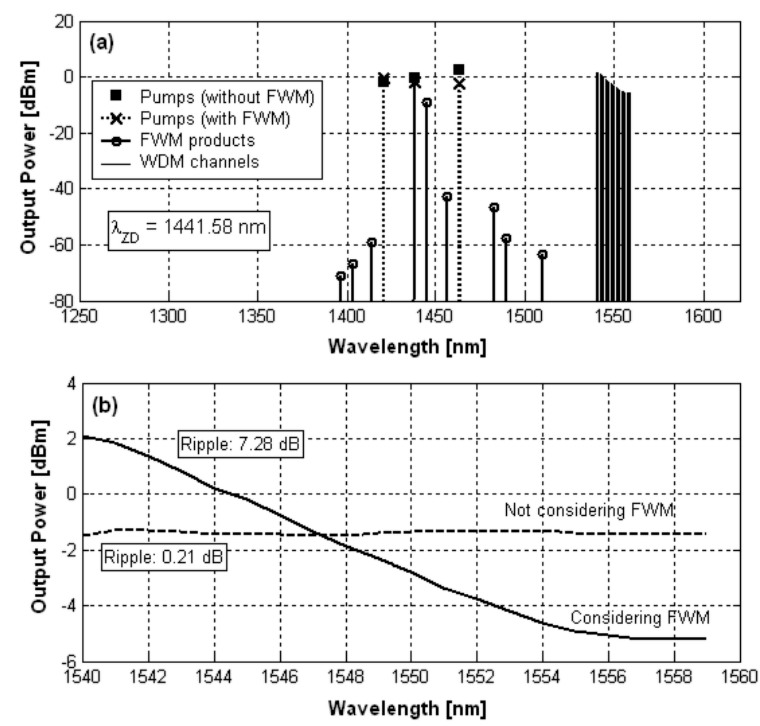

Figure 6. Output power of DFRA\#1, when $\lambda_{\mathrm{ZD}}=1441.58 \mathrm{~nm}$. (a) Output spectrum, including pumps, FWM products, and channels. (b) Output power for WDM channels (with and without including FWM and parametric amplification processes).

and the obtained spectrum including parametric gain of the pumps and pump-pump FWM processes. It can be noted that $\sim 7.0 \mathrm{~dB}$ of ripple is obtained when FWM is undertaken.

\subsection{Analysis of DFRA\#3}

In DFRA\#3, the Raman pumps are spectrally spread in a band of $\sim 85 \mathrm{~nm}$; hence, FWM products are also generated in a wide bandwidth. Similar to previous systems, in this amplifier there are several positions of $\lambda_{Z D}$ which will generate efficient FWM processes. However, when the $\lambda_{\mathrm{ZD}}$ is near the longest pump wavelengths, the most efficient FWM products will be strongly amplified by SRS, mainly because of the broadband characteristic of the DFRA. For instance, when $\lambda_{\mathrm{ZD}}=1490.05 \mathrm{~nm}$, five strong FWM components are generated at the longest wavelengths. Those FWM lightwaves grow from noise at the fiber input, achieving a maximum intensity at a distance equal to the coherence length. Then, they are amplified by SRS with a gain depending on their wavelengths, as shown in figures 9(a) and (b). The main drawback is that these FWM components are generated within the WDM band, as shown in figures 9(b) and (c). Moreover, the intense Raman amplification of the mentioned FWM signals produces an additional depletion of the pumps. However, the pump at $1490.05 \mathrm{~nm}$ results to be the most affected one, mainly because of the depletion caused by the exchange of power in FWM processes. Meanwhile, pumps with shorter wavelength are affected just by a small depletion. As consequence, the net gain of the DFRA is reduced by $2.5 \mathrm{~dB}$ for channels at the longest wavelengths.

\section{Conclusions}

In this work, we have rigorously analyzed the interplay between SRS and FWM in DFRAs by using a novel and 

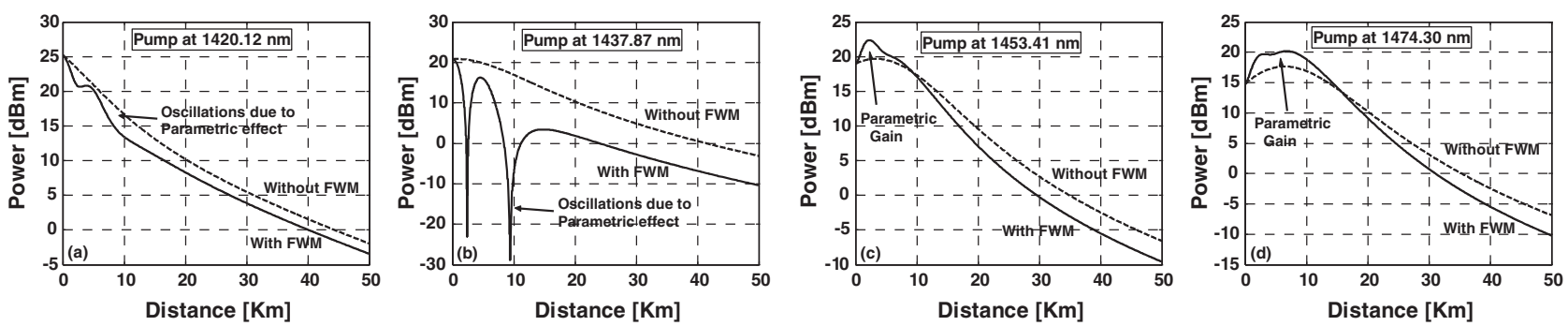

Figure 7. Longitudinal propagation of pump power on DFRA\#2, when $\lambda_{\mathrm{ZD}}=1428.94 \mathrm{~nm}$. (a) Pump at $1420.12 \mathrm{~nm}$. (b) Pump at $1437.87 \mathrm{~nm}$. (c) Pump at $1453.41 \mathrm{~nm}$. (d) Pump at $1474.30 \mathrm{~nm}$.
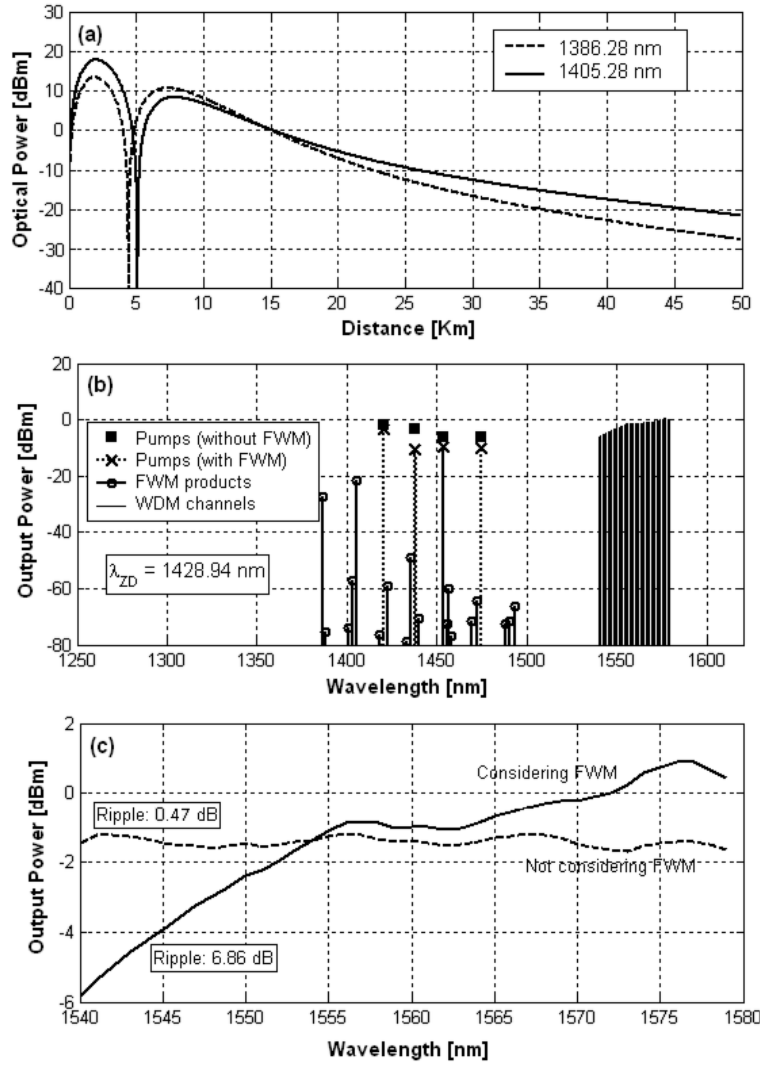

Figure 8. Output power of DFRA\#2, when $\lambda_{\text {ZD }}=1428.94 \mathrm{~nm}$. (a) Most efficient FWM signals. (b) Output spectrum, including pumps, FWM products, and channels. (c) Output power for WDM channels (with and without including FWM and parametric amplification processes).

comprehensive mathematical model. Numerical simulations agree with experimental results, showing the presence of pump-pump FWM in a DFRA implemented in a fiber with low chromatic dispersion. Numerical results show that new components resulting from pump-pump FWM processes act as new pumping sources in the amplifier. Thus, FWM processes and parametric gain produce a spectral and longitudinal redistribution of the Raman pump power. This can produce degradation in the performance of the amplifier such as loss of flatness on the spectral gain, reduction on the net Raman gain, and the presence of strong FWM products within the transmission band. The power exchange between pumps
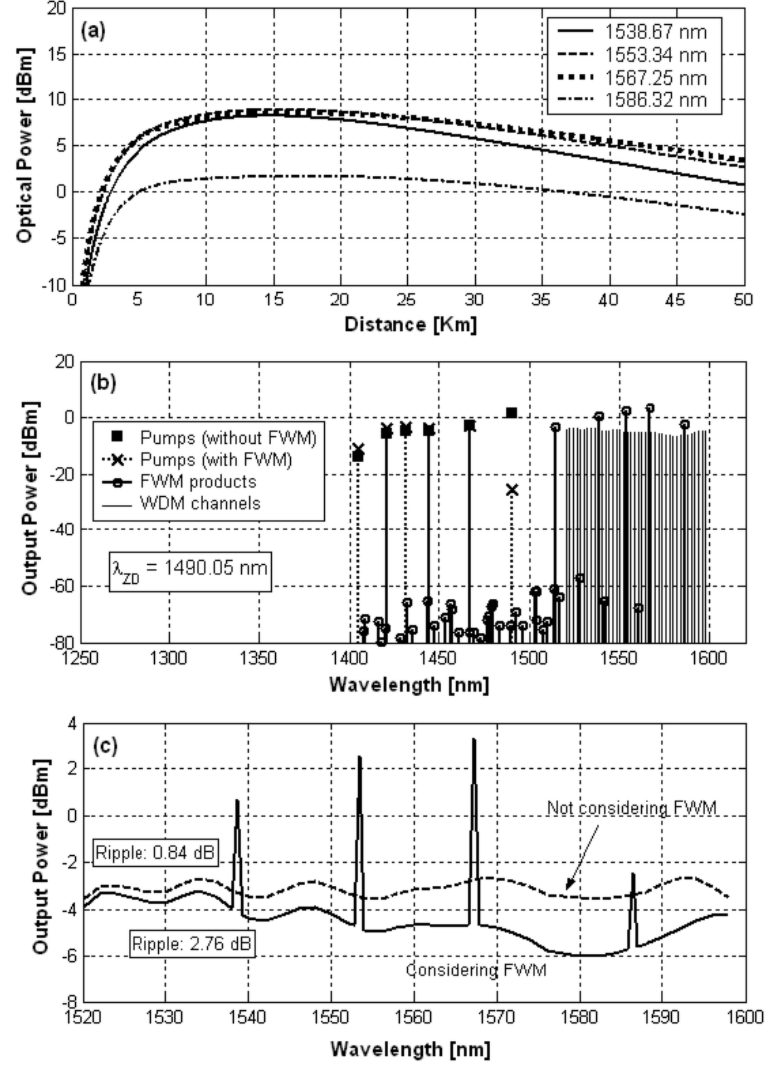

Figure 9. Output power of DFRA\#3, when $\lambda_{\mathrm{ZD}}=1490.05 \mathrm{~nm}$. (a) Most efficient FWM signals. (b) Output spectrum, including pumps, FWM products, and channels. (c) Output power for WDM channels (with and without including FWM and parametric amplification processes).

and FWM products has an oscillatory behavior along the fiber, depending on the position of the $\lambda_{\mathrm{ZD}}$. Therefore, the localization of $\lambda_{\mathrm{ZD}}$ with respect to pump frequency range is a crucial factor in the occurrence of impairments in a gainequalized DFRA.

We have verified that when $\lambda_{\text {ZD }}$ coincides with one of the largest pump wavelengths, an additional depletion of pumps occurs due to Raman amplification of the most efficient FWM components, which are generated in wavelengths longer than those of the pumps. As a consequence, the net gain of the amplifier is reduced. For broadband amplified systems in which $\lambda_{\mathrm{ZD}}$ is close to the longest pump wavelengths, efficient 
FWM components can be generated within the transmission band, interfering with several channels of the system and degrading the OSNR.

On the other hand, when $\lambda_{Z D}$ is located near the shortest pump wavelengths, or near the middle of the pumping band, efficient FWM can produce a redistribution of pump power affecting the gain equalization of the system.

It is possible to conclude that FWM processes, including depletion and parametric amplification, must be taken into account when designing gain-equalized DFRAs pumped at multiple wavelengths, in order to ensure an expected performance.

\section{Acknowledgments}

The authors would like to thank the Chilean Agency CONICYT (Fondecyt project No. 1010437) and the Universidad Técnica Federico Santa María (DGIP project No. 230847) for their financial support of this work.

\section{References}

[1] Bromage J 2004 Raman amplification for fiber communications systems J. Lightwave Technol. 22 79-93

[2] Kidorf H, Rottwitt K, Nissov M, Ma M and Rabarijaona E 1999 Pump interactions in a $100 \mathrm{~nm}$ bandwidth Raman amplifier IEEE Photon Technol. Lett. 11 530-2

[3] Perlin V E and Winful H G 2002 Optimal design of flat-gain wide-band fiber Raman amplifier J. Lightwave Technol. 20 250-4

[4] Chen J, Liu X, Lu C, Wang Y and Li Z 2006 Design of multistage gain-flattened fiber Raman amplifiers J. Lightwave Technol. 24 935-44

[5] Soto H, Pincheira V and Olivares R 2004 Optimal design based on genetic algorithm of distributed fiber Raman amplifier cascades 5th Iberoamerican Mtg on Optics and 8th Latin
American Meeting on Optics, Lasers, and their Applications; Proc. SPIE 5622 364-7

[6] Cui S, Liu J and Ma X 2004 A novel efficient optimal design method for gain-flattened multiwavelength pumped fiber Raman amplifier IEEE Photon Technol. Lett. 16 2451-3

[7] Hill K O, Johnson D C, Kawasaki B and MacDonald R I 1978 $\mathrm{Cw}$ three-wave mixing in single-mode optical fibers J. Appl. Phys. 49 5098-106

[8] Shibata N, Braun R and Waarts R 1987 Phase-mismatch dependence of efficiency of wave generation through four-wave mixing in a single-mode optical fiber IEEE $J$. Quantum Electron. 23 1205-10

[9] Inoue K 1992 Four-wave mixing in an optical fiber in the zero-dispersion wavelength region J. Lightwave Technol. $101553-61$

[10] Wong W S, Chen C-J, Ho M-C and Lee H K 2003 Phase-matched four-wave mixing between pumps and signals in a copumped Raman amplifier IEEE Photon. Technol. Lett. 15 209-11

[11] Bouteiller J-C, Leng L and Headley C 2004 Pump-pump four-wave mixing in distributed Raman amplified systems J. Lightwave Technol. 22 723-32

[12] Vanholsbeeck F, Coen S, Emplit P, Haelterman M and Sylvestre T 2005 Coupled-mode analysis of stimulated Raman scattering and four-wave mixing in wavelength-division multiplexed systems Opt. Commun. 250 191-201

[13] Hansryd J, Andrekson P A, Westlund M, Li J and Hedekvist P-O 2002 Fiber-based optical parametric amplifiers and their applications IEEE J. Sel. Top. Quantum Electron. 8 506-20

[14] Inoue K 1992 Polarization effect on four-wave mixing efficiency in a single-mode fiber IEEE J. Quantum Electron. 28 883-94

[15] Hart D L, Judy A F, Roy R and Beletic J W 1998 Dynamical evolution of multiple four-wave-mixing processes in an optical fiber Phys. Rev. E 57 4757-74

[16] Grant A R 2002 Calculating the Raman pump distribution to achieve minimum gain ripple IEEE J. Quantum Electron. 38 1503-9 